\title{
A Symptomless Carrier of Disease in Helminthosporium victoriae
}

\author{
By G. D. LINDBERG \\ Department of Botany and Plant Pathology, Louisiana State University, \\ Agricultural Experiment Station, Baton Rouge, Louisiana, U.S.A.
}

(Accepted for publication I4 August 1967)

\begin{abstract}
SUMMARY
A mutant (DM-I A) obtained from colonies of a diseased isolate (D-I A) of Helminthosporium victoriae carried the causal agent of disease but showed almost no symptoms. Typical disease symptoms were produced in healthy $H$. victoriae after hyphal contact with the diseased mutant (DM-I A). Hence, the character responsible for suppression of severe disease symptoms in the mutant did not appear to be transmitted with the agent of disease. Healthy mutant cultures that did not carry the causal agent of disease were obtained by isolation of hyphal tips from the diseased mutant. Such cultures inoculated with a second diseased isolate (D-IB) remained nearly symptomless but were capable of transmitting the disease to healthy cultures. The diseased mutant (DM-I A) inoculated with diseased isolate D-I B usually transmitted only D-I A to healthy cultures and the diseased mutant (DM-IB) inoculated with D-I A usually transmitted only D-I B to healthy cultures. This indicates that the two agents tend to be mutually exclusive.
\end{abstract}

\section{INTRODUCTION}

In the course of cultivation of diseased isolate D-I A of Helminthosporium victoriae M. \& M. a mutant was observed and isolated. The mutant had a rate of growth approximately equal to that of healthy $H$. victoriae but had cultural characteristics markedly different from those of the healthy. Preliminary investigations have indicated that the mutant contained the causal agent of disease even though it showed no severe symptoms of disease (Lindberg, 1960). This investigation was undertaken to determine whether the absence of severe symptoms in the diseased mutant was due to a change in the causal agent of disease or whether the mutant behaved as a symptomless carrier of the causal agent. It was considered possible also that investigations utilizing the mutant might elucidate other characteristics of the disease in $H$. victoriae.

\section{METHODS}

A description of the two diseased isolates of Helminthosporium victoriae used in this investigation has been published (Lindberg, I966). The diseased isolates were distinguished on the basis of symptoms and were designated D-I A and D-I B. Strain SN-I of $H$. victoriae was the healthy fungus used throughout this investigation. The mutant isolated from diseased colonies of D-I A contained the causal agent of that disease and was designated DM-I A. Mutant cultures freed of the agent of disease by hyphal tip isolation were called healthy mutants. The mutant cultures that contained the agent of disease D-I B were designated DM-I B. 
Two methods were used in attempts to transfer the disease agent to healthy fungus. The first, called 'hyphal contact inoculation', consisted of placing mycelial seedpieces of the diseased fungus and the fungus to be inoculated approximately $\mathrm{I} \mathrm{cm}$. apart in culture plates. In the second, called 'dip inoculation', cubes of mycelial mat plus agar of healthy $H$. victoriae were dipped into suspensions that contained viable fragments of the diseased fungus. Type symptoms of diseased isolates D-I A and D-I B were best expressed by the dip inoculation method. This method was often used to confirm the disease type after transmission by the hyphal contact method of inoculation.

\section{RESULTS}

The mutant (DM-IA) isolated from colonies of diseased isolate D-I A of Helminthosporium victoriae had a rate of growth approximately equal to that of the healthy fungus. The mutant, unlike the healthy fungus, however, sporulated very sparsely and had other characteristics of growth markedly different from those of the healthy fungus. Colonies of the diseased mutant (DM- I A) were dark grey in colour and the only apparent symptom of disease was the premature collapse of aerial mycelium (lower colony Pl. I, fig. I B). The mutant was observed in diseased colonies of D-I A each year during the spring, but was never observed until the room temperature reached $28^{\circ}$ or above. The mutant has never been observed during the winter at room temperatures of $23-26^{\circ}$ or during the summer when cultures of diseased isolate D-I A were incubated at artificial temperatures of $23^{-}-26^{\circ}$. The mutant has never been observed in cultures of diseased isolate D-I B or in any cultures of healthy $\mathrm{H}$. victoriae. The mutant has been maintained on culture plates or tubes of potato dextrose agar (PDA) for several years without having shown any signs of reverting to the diseased type growth. Mutant cultures were maintained continuously at room temperature $\left(23-3 r^{\circ}\right)$.

\section{Test for the agent of disease in the mutant}

Since the mutant was obtained from diseased fungus it was of interest to determine whether or not it carried the causal agent of disease. Pieces of mycelial mat of the mutant (DM-I A) and of healthy Helminthosporium victoriae (SN-I) were placed approximately I cm. part in culture plates of PDA. Hyphae of the mutant and SN-I touched after approximately $24-36 \mathrm{hr}$ of growth. In 10 culture plates in each of the four experiments, symptoms identical to those of diseased isolate D-I A developed in all colonies of SN-I (upper colony, Pl. I, fig. I B).

Even though the causal agent of disease was present in the mutant (DM-IA), severe diseased symptoms typical of diseased isolate D-I A were not expressed. It became of interest to determine whether the factor responsible for suppression of severe disease symptoms in DM-I A could be transmitted to SN-I along with the causal agent of disease. Following hyphal contact between DM-I A and SN-I, portions of the colony margins of the latter which developed symptoms of disease were removed, ground in a mortar and diluted with sterile water. Small volumes of the suspension were placed on PDA plates with a wire loop. About 20-25 loops of the suspension, spaced approximately $\mathrm{I}-2 \mathrm{~cm}$. apart, were placed in each culture plate (Pl. I, fig. 2). There were over 1500 colonies in 5 experiments and all developed symptoms identical to those of diseased isolate D-I A. There was no suppression of severe diseased symptoms in any of the colonies nor was the mutant type growth ever detected. 


\section{Hyphal tip isolation of the normal mutant}

The most severe symptoms in colonies of D-I A occurred in the young cells at the colony margin (Lindberg, 1959). Such cells lysed and the colonies showed little further radial growth (Psarros \& Lindberg, 1962). Hyphal tips isolated from D-I A usually failed to grow but occasionally produced small diseased colonies.

The mycelial collapse characteristic of DM-I A occurred first in the oldest portion or centre of the colony and gradually spread outward. The young hyphal cells at the colony margin appeared unaffected and continued to grow. Therefore, an attempt was made to obtain the mutant free of the causal agent of disease by isolation of hyphal tips. Twenty hyphal tips were isolated from 36- to 48-hr-old colonies of DM-I A in each of four experiments. Fifty-five of the colonies showed no collapsed aerial mycelium and were tentatively classified as healthy mutants. Twenty-five of the colonies showed collapsed aerial mycelium and were classed as diseased (DM-I A). Twenty-six of the colonies classified as healthy mutants were tested for the agent of disease by hyphal contact inoculation of SN-I. Symptoms of disease failed to develop in any of the inoculated $\mathrm{SN}-\mathrm{I}$ colonies and indicated that the mutant colonies classified as healthy did not contain the agent of disease. Eleven of the colonies classified as DM-I A were tested against SN-I and symptoms identical to those of D-I A developed in all of the inoculated $\mathrm{SN}-\mathrm{I}$ colonies.

\section{Inoculation of the healthy mutant with $D-I B$}

A mutant similar to the one isolated from D-I A has never been observed in colonies of D-I B. Since healthy mutant cultures were obtained by isolation of hyphal tips of DM-I A, it was of interest to determine what reaction the healthy mutant might have to inoculation with D-I B. It was considered possible that the agent of diseased isolate D-I B might produce more severe symptoms in the mutant than those produced by the agent of diseased isolate D-I A. Ten culture plates of PDA were seeded with the healthy mutant and D-I B in each of 4 experiments. Four or 5 days after hyphal contact with D-I B the once-healthy mutant was subcultured. The colonies that developed had a light grey colour and the aerial hyphae collapsed only slightly (lower colony, Pl. I, fig. I A). Such colonies were then tested for diseased type by hyphal contact inoculation of healthy Helminthosporium victoriae (SN-I). In Io PDA plates in each of 4 experiments, diseased symptoms identical to those of diseased isolate D-I B developed in the $\mathrm{SN}-\mathrm{I}$ test colonies of all 40 plates (upper colony, Pl. I, fig. I A). Diseased mutant colonies that contained the agent of disease D-I B were designated DM-I B. It should also be stated that healthy mutant cultures inoculated with D-I A were readily infected and transmitted only D-I A to healthy $H$. victoriae.

\section{Protection studies}

It then became of interest to determine whether or not a mixture of the agents of the diseases, D-I A and D-I B, could be established in the mutant. If the agents of both diseases were present the mutant might show a synergistic reaction. The presence of one agent of disease in the mutant might, on the other hand, protect against infection by the other agent of disease.

To test these possibilities DM-I A was confronted with inoculum of D-I B and DM-I B was confronted with inoculum of D-IA. Ten of each of the above pairings were made in culture plates of PDA in each of 5 experiments. DM-I A and DM-I B challenged with 
D-I B or D-IA, respectively, showed no change in symptoms. Nevertheless, pieces of the mycelial mats of the challenged DM-IA and DM-I B were subcultured on PDA plates. All subcultures of the DM-I A appeared identical to the type colonies of DM-I A and all subcultures of the DM-I B appeared identical to the type colonies of DM-IB.

The subcultures were then tested for disease type by hyphal contact inoculation of SN-I. Of 50 test colonies of SN-I confronted with mycelium of the subcultures of challenged DM-I A, 44 developed symptoms of D-I A and 6 developed symptoms of D-I B. Of 50 colonies of SN-I confronted with mycelium of the subcultures of challenged DM-I B, 45 developed symptoms of D-I B and 5 colonies developed symptoms of D-I A (Table I).

Table I. DM-IA and DM-IB were inoculated by hyphal contact with $D-I A$ and $D-I B$, respectively, and subcultured: the subcultures were tested for disease type against healthy Helminthosporium victoriae $(S N-I)$

\begin{tabular}{|c|c|c|c|c|c|}
\hline \multirow[b]{3}{*}{ Experiment } & \multirow{3}{*}{$\begin{array}{c}\text { Date } \\
\text { (I966) }\end{array}$} & \multicolumn{4}{|c|}{ Diseased type transmitted to $\mathrm{SN}-\mathrm{I}$} \\
\hline & & \multicolumn{2}{|c|}{$D M-I A / D-I B$} & \multicolumn{2}{|c|}{$D M-I B / D-I A$} \\
\hline & & D-I A & D-I B & D-I A & D-I B \\
\hline I & 6 Jan. & $10 / 10$ & $0 / 10$ & $0 / 10$ & $10 / 10$ \\
\hline 2 & 22 Jan. & $10 / 10$ & $0 / 10$ & $0 / 10$ & $10 / 10$ \\
\hline 3 & I5 Mar. & $10 / 10$ & $0 / 10$ & $5 / 10$ & $5 / 10$ \\
\hline 4 & 25 Mar. & $10 / 10$ & $0 / 10$ & $0 / 10$ & $10 / 10$ \\
\hline 5 & 9 May & $4 / 10$ & $6 / 10$ & $0 / 10$ & $10 / 10$ \\
\hline
\end{tabular}

\section{DISCUSSION}

Except for the premature collapse of aerial mycelium DM-I A and DM-I B could be considered symptomless carriers.

The symptoms of disease that developed in healthy Helminthosporium victoriae (SN-I) inoculated by hyphal contact with DM-I A were identical to those of diseased isolate D-I A. This indicated that the absence of severe symptoms in DM-IA was not due to a change in the agent of disease and that the character responsible for suppression of severe disease symptoms resided with the mutant.

Jinks (1964) has shown that when a normal homokaryon and a vegetative death variant of Aspergillus glaucus are grown side by side, heterokaryon sectors formed between them, but beyond these sectors ever widening sectors with the vegetative death phenotype replaced the normal homokaryon. Since the vegetative death phenotype penetrated well beyond the maximum point of penetration of the known chromosomally borne determinants, Jinks concluded that an unambiguous criterion of extrachromosomal heredity had been satisfied. The phenomenon was referred to as 'infective heredity'. Assays of diseased mycelium that developed in Helminthosporium victoriae inoculated by hyphal contact with the diseased mutant (DM-IA) indicated that the factor responsible for suppression of severe diseased symptoms was not transmitted with the causal agent of disease. If the factor responsible for the mutant type of growth is controlled in the nucleus it seems likely that the agent of disease might be extranuclear or cytoplasmic.

DM-IA inoculated by hyphal contact with D-IB was subcultured and tested for disease type against SN-I. The SN-I test colonies usually developed symptoms identical 

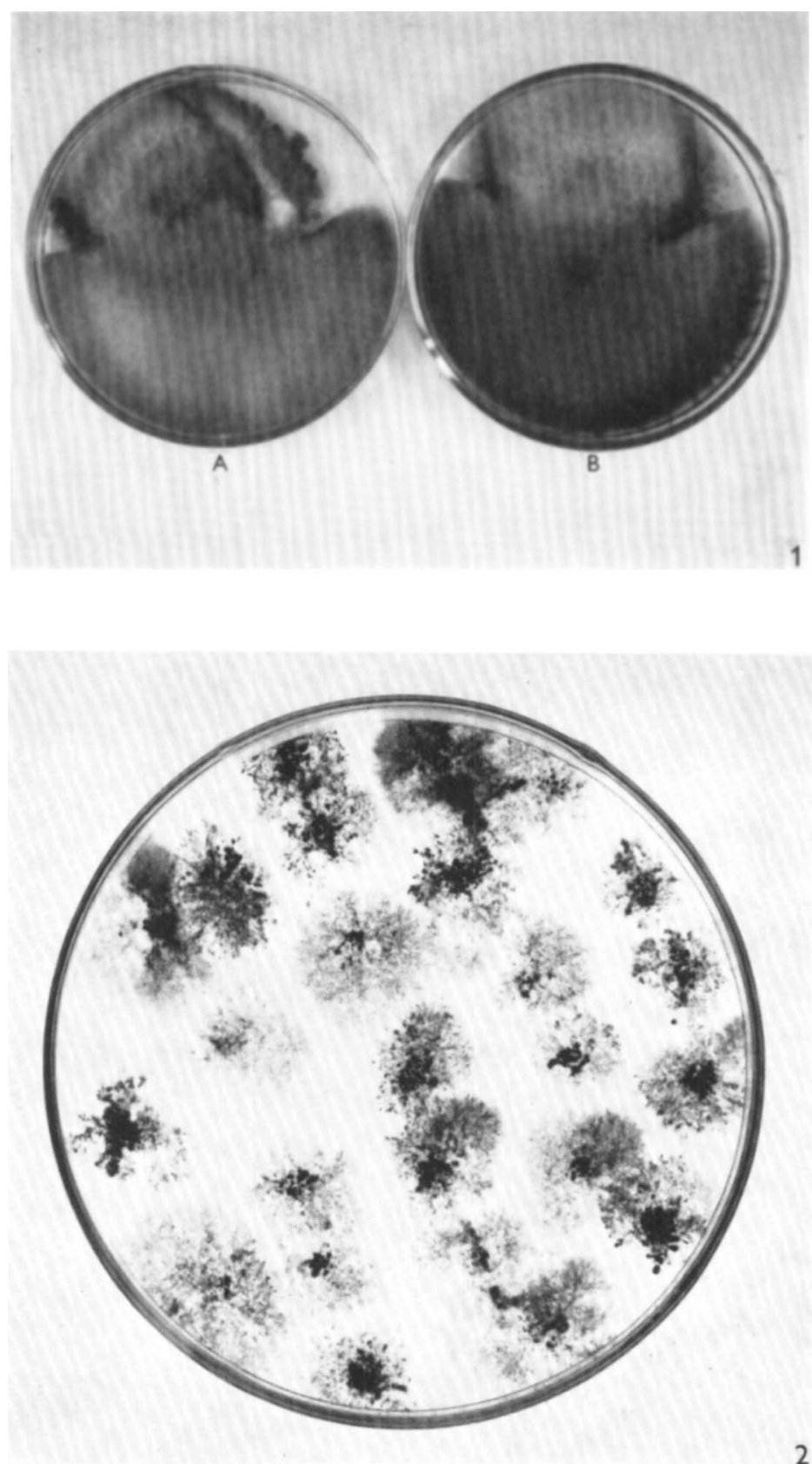

G. D. LINDBERG

(Facing p. 365) 
to those of diseased isolate D-I A. DM-I B inoculated by hyphal contact with D-I A was subcultured and tested for disease type against SN-I. The SN-I test colonies usually developed symptoms identical to those of diseased isolate D-I B. These results indicated that the presence of the agent of disease D-I A may have protected the mutant against infection by the agent of disease D-I B and vice versa. The protection, if indeed that was the case, was not complete.

This work was supported in part by National Institutes of Health (U.S.A.) grant no. I A-04700.

\section{REFERENCES}

JINKS, J. L. (I964). Extrachromosomal Inheritance. Englewood Cliffs, N.J., U.S.A.: Prentice-Hall. LindBerg, G. D. (1959). A transmissible disease of Helminthosporium victoriae. Phytopathology 49, 29.

Lindberg, G. D. (I 960). Transmission of a disease of Helminthosporium victoriae. Phytopathology 50 , 644.

LindBerg, G. D. (I966). Production of disease in Helminthosporium victoriae with phenol extracts of diseased and normal mycelia. Phytopathology 56, 1297.

Psarros, E. E. \& LindberG, G. D. (1962). Morphology and respiration of diseased and normal Helminthosporium victoriae. Phytopathology 52, 693.

\section{EXPLANATION OF PLATE}

Fig. 1. A. Diseased mutant (DM-IB, lower colony) originally plated with healthy Helminthosporium victoriae (SN-I). Diseased symptoms identical to those of diseased isolate D-IB developed in the latter (upper colony). B. Diseased mutant (DM-I A, lower colony) originally plated with healthy $H$. victoriae. Diseased symptoms of D-I A developed in the latter (upper colony).

Fig. 2. Colonies are from Helminthosporium victoriae that developed symptoms of disease following hyphal contact with the diseased mutant (DM-I A). The colonies show symptoms of disease D-I A but the factor responsible for suppression of disease symptoms in the mutant was not detected. 